\title{
Análise, Revisão e Aplicação da Abordagem para Inclusão do Licenciado em Computação no Ensino Básico (ABILSEN)
}

\author{
Máverick André D. Ferreira ${ }^{1}$, Débora da C. Araújo ${ }^{1}$, Alisson P. da Silva ${ }^{1}$, \\ Sebastião S. Neto ${ }^{1}$, Higor M. Santos ${ }^{1}$, Célia C. Silva ${ }^{1}$, Ariane Nunes Rodrigues ${ }^{1}$ \\ ${ }^{1}$ Universidade de Pernambuco (UPE) \\ CEP 55.294.902 - Garanhuns - PE - Brazil \\ \{amaverick70, deeboraaraaujo, alissonnpereira, sebast.rogers, \\ higormonteiro1206, celinhacristina20, ariane.upe\}@gmail.com
}

\begin{abstract}
Contemporary society requires its professional skills that are not stimulated by the school. To encourage these skills, it becomes apparent the need for adherence to the teaching of other sciences also in Basic Education, the example, the Computer Science. In this sense, the degree courses in Computer are designed to train professionals that met this new educational demand. Thus, this research portrays an analysis and application of the approach to Bachelor of Inclusion in Computer Science in Basic Education (ABILSEN). The results show that ABILSEN can contribute to computer education, interdisciplinary, considering the current curricular context.
\end{abstract}

Resumo. A sociedade contemporânea exige das pessoas algumas habilidades que não são estimuladas pela escola. Para estimular essas habilidades, tornase perceptivel a necessidade da adesão ao ensino de outras ciências ainda na Educação Básica, à exemplo, a Ciência da Computação. Nesse sentido, os cursos de Licenciatura em Computação foram criados para formar profissionais que atendessem essa nova demanda educacional. A presente pesquisa retrata uma análise, revisão e aplicação da Abordagem para Inclusão do Licenciado em Computação no Ensino Básico (ABILSEN). Os resultados obtidos demonstram que a ABILSEN pode contribuir com o ensino de computação, de forma interdisciplinar, considerando o atual contexto curricular.

\section{Introdução}

A criança imersa, cada vez mais cedo, em um cenário rodeado de tecnologias passa a não se contentar apenas com uso das ferramentas disponíveis, mas também em criar suas próprias ferramentas, como pode ser percebido por meio de algumas iniciativas à exemplo do HappyCode ${ }^{1}$. Além do interesse e curiosidade inerentes do nativo digital pelas Tecnologias da Informação e Comunicação (TIC), muitos são os fatores que apontam para a necessidade de se ter o ensino de Computação desde a Educação Básica. Dentre esses fatores, destacam-se estudos que direcionam para um crescimento na demanda de profissionais da área de tecnologia até o ano de 2018, bem como a escassez de profissionais qualificados (Scaico et al. 2012).

\footnotetext{
${ }^{1}$ Disponível em: http://www.happycode.com.br/games-atraem-criancas-para-a-programacao/
} 
Além disso, o ensino de Computação, enquanto ciência, é indicado até mesmo para profissionais que não se interessam pelo mercado tecnológico, tendo em vista que o estudo de lógica de programação pode proporcionar o desenvolvimento de habilidades desejáveis para o profissional do século XXI (Araújo et al. 2015). Dessa forma, para atender a essa nova demanda da sociedade e promover o ensino de Computação nos diversos níveis de educação, foram criados os cursos de Licenciatura em Computação. Neto et al. (2015), reforçam ainda que esses cursos formam profissionais com a capacidade tanto de desenvolver programas/sistemas, quanto de analisar e aplicar tecnologias de modo a contribuir para o processo de ensino-aprendizagem nos níveis Básico, Técnico e Profissionalizante.

Com isso, dois problemas macro podem ser identificados no contexto brasileiro: a ausência de uma disciplina que trate dos conceitos e técnicas da Ciência da Computação ainda na Educação Básica e a necessidade de atentar-se para estratégias de ensino que propiciem um melhor engajamento dos educandos durante o processo de ensino-aprendizagem. Nessa perspectiva, a ludicidade se apresenta como um importante instrumento, tendo em vista sua capacidade de possibilitar a realização de atividades de maneiras despretensiosas e descontraídas (SÁ, 2004). Ao traçar um paralelo entre o lúdico, a realidade dos estudantes e a importância de se ensinar conceitos de computação desde a educação básica coloca-se em discussão a utilização e criação de jogos eletrônicos nas salas de aula.

Nesse contexto, Neto et al. (2015) propuseram a Abordagem para Inclusão do Licenciado em Computação no Ensino Básico (ABILSEN), que objetiva inserir a Computação no ensino Básico de forma lúdica e interdisciplinar às demais disciplinas presentes no currículo. Mesmo com os resultados positivos obtidos com a aplicação da ABILSEN, em Neto et al. (2015), foi possível observar algumas possibilidades de melhorias para a abordagem. Diante disso, o presente trabalho tem como objetivo relatar a análise, revisão e aplicação da ABILSEN em um novo contexto, uma turma de $9^{\circ}$ ano do ensino fundamental. Para tanto, o artigo foi organizado da seguinte forma: a Seção 2 descreve as principais características da ABILSEN, além de alguns trabalhos relacionados; a Seção 3 faz uma análise e posterior revisão da ABILSEN com base nos relatos de Neto et al. (2015); a Seção 4 descreve os resultados obtidos com a nova aplicação; e a Seção 5 relata as considerações finais.

\section{Abordagem (ABILSEN)}

A ABILSEN, proposta em Neto et al. (2015), é baseada no ciclo PDCA (Planejar, Executar, Verificar e Refinar). O ciclo de atividades proposto para a abordagem possibilita aos licenciados em Computação a experiência de planejar aulas em conjunto com professores de outras áreas, participar da execução dessas aulas (não apenas como suporte), avaliar a aprendizagem dos educandos e discutir sobre as práticas adotadas objetivando uma melhoria contínua.

A ABILSEN foi organizada em 7 etapas: Definição do Ambiente (DA) escolha do ambiente (escola) e/ou disciplina onde será aplicada a abordagem levando em consideração os recursos tecnológicos da escola (computadores, internet e softwares); Planejamento da Disciplina (PD) - planejamento da disciplina participante (Português, Matemática etc.) em conjunto com o professor da mesma, explorando o potencial em 
V Congresso Brasileiro de Informática na Educação (CBIE 2016)

Anais do XXII Workshop de Informática na Escola (WIE 2016)

termos de interdisciplinaridade de ambas as áreas; Seleção de Objetos de Aprendizagem Eletrônicos (SOE) - seleção e instalação de OA eletrônicos que contemplem os assuntos que deverão ser abordados na disciplina em questão; Aplicação com Mediação (AM) condução dos alunos para o laboratório de informática para aplicar os OA selecionados em conjunto com o professor da disciplina; Desenvolvimento do Objeto de Aprendizagem (DOA) - ensino de conceitos básicos de programação aos alunos para que eles produzam Objetos de Aprendizagem relacionados aos assuntos abordados na disciplina em questão; Avaliação da Aprendizagem (AA) - avalia os benefícios gerados aos estudantes e procurar listar possíveis melhorias para as próximas aplicações. Refinamento das Práticas (RP) análise da execução com base na eficiência e eficácia da abordagem de modo a identificar pontos positivos e negativos.

Apesar desta pesquisa tomar como base Neto et al. (2015), também levaram-se em consideração outros trabalhos publicados na literatura com direcionamento tanto para o ensino de Computação na educação básica, como o ensino baseado em gamificação. Em Fioro et al. (2014) é relatado um estudo que analisa a mudança de comportamento apresentada por alunos devido a utilização da robótica durante o ensino de Matemática, Física e Química. Castro e Fernandes (2013) apresentam um ambiente gamificado para o ensino de Química que explora elementos presentes nos games, tais como ranking, recompensa entre outros.

\section{Métodos}

Em Neto et al. (2015), a ABILSEN é colocada como uma abordagem passível de replicação em outros momentos/espaços. Dessa forma, esta pesquisa foi dividida em três fases: Análise da ABILSEN - nesta fase buscou-se investigar as possibilidades de melhorias na estrutura da ABILSEN levando em consideração a sua facilidade de replicação. Revisão da ABILSEN - nesta fase o objetivo foi implementar as melhorias identificadas na fase anterior de modo a tornar mais fácil e eficaz a aplicação da abordagem. Aplicação da ABILSEN - esta fase proporcionou uma nova rodada de aplicação da abordagem com base nas melhorias sugeridas na fase de Revisão.

Esta pesquisa foi conduzida por um professor, quatro alunos e um egresso do curso de Licenciatura em Computação. Todos os pesquisadores envolvidos participaram das fases de Análise e Revisão. E um dos licenciandos e o egresso realizaram a fase de Aplicação da ABILSEN. Ao analisar as etapas da abordagem proposta, em Neto et al. (2015), algumas lacunas foram identificadas com relação a como executá-la, são elas: (DA) - Quais características deve ter a instituição de ensino analisada para que seja considerada apta para realização da abordagem? (PD) - Quais pontos devem nortear a discussão com o professor da disciplina selecionada? (SOE) - São levadas em consideração as diferenças de perfis de aprendizagem de cada aluno? (DOA) - Quais os critérios para formação dos grupos de alunos? (RP) - Quais as métricas para "eficiência" e "eficácia" no contexto da ABILSEN?

Com base nessas lacunas, acredita-se que a elaboração de perguntas de cunho reflexivo pode contribuir para que o licenciado em Computação, ao respondê-las, possa delinear critérios que facilitem a execução da ABILSEN. Abaixo são expostas as perguntas propostas para cada etapa: 
V Congresso Brasileiro de Informática na Educação (CBIE 2016)

Anais do XXII Workshop de Informática na Escola (WIE 2016)

- (DA) - É necessário o uso de um computador por aluno? Será necessário que a instituição de ensino tenha acesso à internet? Quais configurações mínimas em termos de hardware suportam os softwares que serão utilizados? Quais sistemas operacionais suportam os softwares que serão utilizados? Qual a melhor técnica para despertar o interesse dos docentes? Quais serão os critérios para seleção das disciplinas?

- (PD) - Como será a organiza a aula expositiva do professor e a utilização dos OA? Quantos objetos de aprendizagem eletrônicos devem ser selecionados para cada disciplina? Quais técnicas podem ser utilizadas para aumentar o engajamento dos alunos na abordagem?

- (SOE) - Quais critérios devem ser levados em consideração durante a seleção dos $\mathrm{OA}$ ?

- (DOA) - Quais os critérios para formação dos grupos de alunos?

- (RP) - Os alunos gostaram da experiência? A abordagem contribuiu para a aprendizagem dos assuntos trabalhados na disciplina em questão? Os alunos conseguiram aprender os conceitos de Computação? Houve algum imprevisto em alguma etapa da abordagem?

A aplicação da ABILSEN com as melhorias propostas pelos autores desta pesquisa ocorreu numa turma de $9^{\circ}$ ano do ensino fundamental de uma escola municipal situada no interior do Estado de Pernambuco. As disciplinas abordadas foram as de Português e Matemática. A turma escolhida era composta por trinta alunos, dos quais dezessete mostraram interesse em participar da abordagem, mas devido à necessidade de se realizar alguns encontros no período vespertino (contra turno), apenas cinco alunos confirmaram presença. Apesar da pequena amostra ser uma ameaça a validade externa, a pesquisa foi realizada como uma forma de oportunidade de recursos. Dessa forma, ressalta-se que os resultados aqui obtidos não são passíveis de ampla generalização.

Ao todo, foram ministradas 15 horas e 20 minutos de aulas para os alunos envolvidos. Sendo 1 hora e 40 minutos (duas aulas com duração de 50 minutos cada) sobre relações trigonométricas, 1 hora e 40 minutos (duas aulas com duração de 50 minutos cada) sobre pronomes demonstrativos e 12 horas para ensino de conceitos introdutórios de Computação para desenvolvimento dos Objetos de Aprendizagem Eletrônicos. A próxima seção detalha como aconteceu a nova aplicação da abordagem com as mudanças realizadas.

\section{Resultados}

\subsection{Definição do ambiente (DA)}

Com o objetivo de definir o ambiente de aplicação foi efetuada uma visita à escola Municipal Giselda Vieira Belo. Ao analisar o laboratório dessa instituição identificou-se a possibilidade de aplicação da ABILSEN. Com o propósito de motivar os professores a participarem da abordagem foi aplicado um questionário constituído das seguintes questões: (1) Com qual frequência você utiliza dispositivos tecnológicos no seu dia a dia?; (2) Como você classifica o grau de importância das tecnologias para mediar o processo de ensino-aprendizagem?; e (3) Com qual frequência você utiliza dispositivos 
tecnológicos para mediar suas aulas?. As respostas obtidas a partir de alternativas do tipo Likert possibilitaram a preparação de uma apresentação personalizada sobre a abordagem para o corpo docente. E, como resultado, os professores de Português e Matemática se propuseram a participar da abordagem.

\subsection{Planejamento da Disciplina (PD)}

Em um primeiro momento buscou-se identificar quais conteúdos de Português e Matemática seriam trabalhados no decorrer da abordagem. E, com isso, ficaram definidos os seguintes conteúdos: Matemática - Relações trigonométricas e Português Pronomes demonstrativos. Após a identificação dos assuntos, o próximo passo foi planejar as aulas em conjunto com os professores. Como forma de aumentar o engajamento dos alunos foi definido que as etapas (AM) e (DOA) fariam uso de técnicas de gamificação que, por sua vez, consiste na utilização de elementos encontrados nos jogos em outros contextos (LOPES et al. 2015). Para tal, alguns elementos de jogos foram levados em consideração, tais como: (1) narrativa - com a caracterização de missões durante o processo de desenvolvimento dos OA; (2) conquistas - com o recebimento de medalhas à medida que eram identificadas habilidades $^{2}$ definidas a priori e (3) pontuação - com a montagem de um ranking cumulativo com pontuação gerada a partir da troca de medalhas, recebidas durante os encontros, por pontos. Vale ressaltar que ao fim de cada encontro um ranking era atualizado e exibido em um quadro informativo da escola.

\subsection{Seleção de Objetos de Aprendizagem eletrônicos (SOE)}

Nesta etapa, buscou-se por (OA) lúdicos que contemplassem os conteúdos que os professores iriam abordar durante as aulas. A seleção foi dividida em três níveis: (1) Busca por artefatos lúdicos nos repositórios: (RIVED, PROATIVA, BIO, FEB, BNDIGITAL) ${ }^{3}$; (2) Procura por artefatos lúdicos por meio do mecanismo de busca do Google; (3) Procura por quaisquer artefatos educativos que contemplem os assuntos abordados. Conforme os critérios para seleção iniciou-se a busca por OA eletrônicos para ensino de Trigonometria nos repositórios definidos a priori. No entanto, apenas dois objetos foram incluídos na seleção (Sinuca e Transbordando) ${ }^{4}$, sendo ativado o segundo nível de busca que implicou na adição de mais um objeto à lista (Tangrans) ${ }^{4}$. Para selecionar os OA para mediar as aulas de Pronomes Demonstrativos adotou-se o mesmo método de busca. E, com isso, no primeiro e segundo nível nenhum objeto foi adicionado. Devido à ausência de OA com características lúdicas, o nível três de procura foi ativado e, com isso, dois artefatos educativos foram selecionados: Pronomes Demonstrativos 1 e Pronomes Demonstrativos 2) ${ }^{4}$. A partir dos resultados obtidos com

\footnotetext{
2 Disponível em: http://migre.me/tjIL2

${ }^{3}$ RIVED: Disponível em: http://rived.mec.gov.br/; PROATIVA: Disponível em: http://www.proativa.vdl.ufc.br/; BIO: Disponível em: http://objetoseducacionais2.mec.gov.br/; FEB: Disponível em: http://feb.ufrgs.br/; BNDIGITAL: Disponível em: https://bndigital.bn.br/

4 Sinuca: Disponível em: http://migre.me/tjIVE; Transbordando: Disponível em: http://migre.me/tjIYE; Tangrans: Disponível em: http:/migre.me/tjJ1x; Pronomes Demonstrativos: Disponível em: http://migre.me/tjJ30; Pronomes Demonstrativos: Disponível em: http://migre.me/tjJ52
} 
a seleção de OA foi possível perceber uma carência por objetos de aprendizagem sobre Pronomes Demonstrativos com características lúdicas.

\subsection{Aplicação com mediação}

Nesta etapa cada professor participante da abordagem ministrou duas aulas com duração de 50 minutos cada. A primeira foi uma aula expositiva e a segunda estimulou a interação dos alunos com os objetos de aprendizagem selecionados para aquele assunto. Devido à ausência de internet na escola em questão, foi utilizado o aplicativo HTTrack ${ }^{5}$ para possibilitar o funcionamento dos OA selecionados em modo offline.

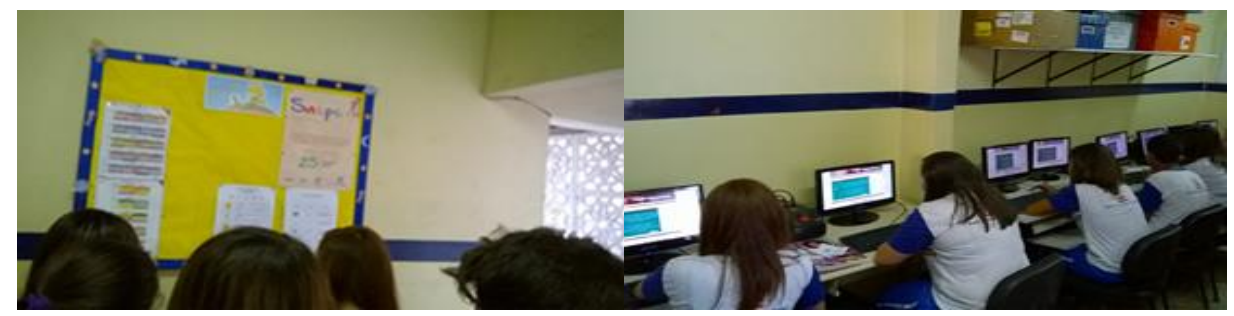

Figura 1. Interação dos estudantes com os OA de Matemática

Ao observar a Figura 1, é possível perceber os alunos interagindo com os OA e verificando o ranking após um dia de atividades.

\subsection{Desenvolvimento de Objetos de Aprendizagem (DOA)}

Nesta etapa, as disciplinas de Português e Matemática foram trabalhadas em conjunto de modo que os alunos desenvolvessem jogos educativos que contemplassem os temas definidos na etapa Planejamento da Disciplina. Para tanto, ocorreram três encontros conduzidos por licenciados em Computação com uma duração de 4h (quatro horas) cada. Ainda, contou-se o com a orientação dos professores de Português e Matemática sobre os assuntos a serem abordados nos OA. Para desenvolver os objetos de aprendizagem foram utilizados os softwares: GameMaker $8.1^{6}$ para codificação do jogo e o Pencil Project para criação das telas.

Esta etapa da aplicação foi subdividida em quatro missões com base no elemento da gamificação denominado narrativa. A primeira missão - Invadindo o território da programação - aconteceu durante o primeiro encontro e a primeira metade do segundo encontro e teve como principal objetivo introduzir conceitos básicos de programação, tais quais: Conceito de algoritmos, Conceito de variáveis, Constantes, Expressões aritméticas e Operadores relacionais, Comandos de atribuição, Estrutura sequencial e Comandos de decisão. A segunda missão - Invadindo o território da criatividade aconteceu durante a segunda metade do segundo encontro e todo o terceiro encontro, objetivando ensinar aos estudantes como criar cenários com o software Pencil Project.

A terceira missão - Fechando alianças - foi destinado ao agrupamento dos participantes em dois grupos de maneira aleatória. Por fim, a quarta missão Construindo um objeto de aprendizagem - teve por objetivo possibilitar aos alunos o desenvolvimento de objetos de aprendizagem, especificamente jogos eletrônicos

\footnotetext{
${ }^{5}$ Disponível em: https://www.httrack.com/.

${ }^{6}$ Disponível em : https://www.yoyogames.com/.
} 
V Congresso Brasileiro de Informática na Educação (CBIE 2016)

Anais do XXII Workshop de Informática na Escola (WIE 2016)

educativos que contemplassem os conteúdos curriculares de Trigonometria e Pronomes Demonstrativos.

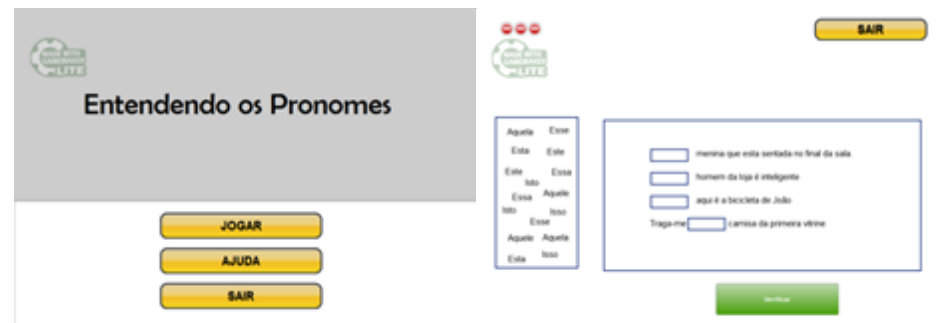

Figura 2. Jogo Entendendo os Pronomes

Os grupos serão referenciados no decorrer do texto como "grupo 1" e "grupo 2". Com os agrupamentos, o "grupo 1" se propôs a desenvolver o jogo "Entendendo os pronomes" e teve como resultado o jogo exibido na Figura 2.

Neste jogo, o usuário deve preencher as lacunas presentes nas frases com o pronome adequado. Ao analisar o game, é possível perceber que, para sua construção, os alunos fizeram uso de alguns conceitos de programação, tais como, Variáveis - Para controlar a quantidade de vidas do jogador e para armazenar os valores referentes aos pronomes inseridos nas lacunas presentes nas frases, Comandos de atribuição - Para decrementar as vidas quando necessário e para inserir os pronomes nas lacunas (variáveis), Comandos de decisão - Para testar se o pronome inserido na lacuna é correto e para testar se o jogador possui vidas. O "grupo 2" produziu o jogo "Labirinto trigonométrico". Neste jogo, a missão é chegar ao quadro roxo, presente no labirinto, como mostra a Figura 3. Para atingir seu objetivo o jogador terá que abrir alguns portais compostos por perguntas sobre relações trigonométricas.

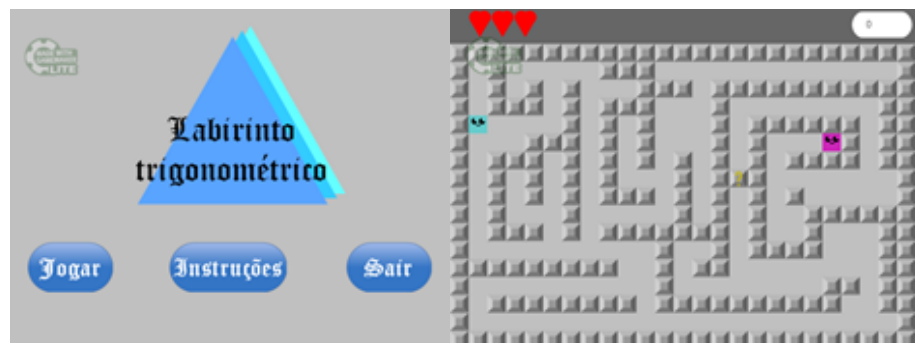

Figura 3. Jogo Labirinto trigonométrico

Ao analisar o jogo produzido pelo grupo 2 identifica-se que os alunos aplicaram Conceitos de variáveis - Para controlar a quantidade de vidas do jogador e para armazenar a pontuação do jogador, Comandos de atribuição - Para decrementar as vidas quando necessário e para incrementar a pontuação dos jogadores, Comandos de decisão - Para testar se a resposta dada pelo jogador está correta e para testar se o jogador possui vidas.

Dado o curto tempo dispendido para o desenvolvimento dos OA, e o fato de ser o primeiro contato dos estudantes participantes com conceitos de programação, acreditase que os resultados foram positivos apesar das características simplistas dos artefatos gerados. $\mathrm{O}$ fato do aluno (participante) ser autor de jogos evidencia a possibilidade do mesmo aprender, de forma prática e divertida, conceitos de Computação, além de possibilitar a aprendizagem de conteúdos curriculares de forma interdisciplinar e 
aplicada. Pois o estudante ao desenvolver um artefato que contemple conteúdos curriculares, tendo como público outros estudantes, estará potencializando sua aprendizagem como menciona Paulo Freire (1994) ao afirma que quem ensina, aprende ao ensinar e quem aprende, ensina ao aprender.

\subsection{Avaliação da aprendizagem}

Essa etapa da abordagem consistiu-se de dois momentos. No primeiro buscou-se identificar os estilos de aprendizagem (EA) dos estudantes por meio dos Índices de Estilos de Aprendizagem de Felder (2002) que contempla 4 (quatro) dimensões de EA: Processamento; estilo Ativo (pessoas que aprendem melhor com experimentação); e estilo Reflexivo (pessoas que preferem dispor de momentos sozinhos para refletirem); Percepção: estilo Sensorial (possuem preferência em lidar com dados concretos); e estilo Intuitivo (lidam melhor com questões abstratas); Entrada: estilo Visual (lembra mais facilmente do que vê em figuras, demonstrações); e Verbal (preferem informações faladas ou escritas); Compreensão: estilo Sequencial (preferem aprender com dificuldade contínua); e estilo Global (aprendem de forma aleatória) (Felder e Silverman, 1988).

Felder (2002) define a seguinte escala para classificar o estilo de aprendizagem dos estudantes: (1-3) mostra que o estudante está bem equilibrado sobre as duas dimensões dessa escala; (5-7) o estudante tem preferência moderada para uma dimensão da escala e vai aprender mais facilmente numa perspectiva que favoreça tal dimensão; (9-11) tem forte preferência por uma das dimensões e, com isso, deverá ter bastante dificuldade em ambientes que não suportem essa preferência. A Tabela 1 exibe os resultados obtidos com a aplicação dos índices.

Tabela 1. Resultado da aplicação dos Índices de Estilos de Aprendizagem

\begin{tabular}{|c|c|c|c|c|c|c|c|c|}
\hline & $\mathbf{9 - 1 1}$ & $\mathbf{5 - 7}$ & $\mathbf{1 - 3}$ & $\mathbf{1 - 3}$ & $\mathbf{5 - 7}$ & $\mathbf{9 - 1 1}$ & \\
\hline Ativo & & 1 & 1 & & 2 & 1 & & Reflexivo \\
\hline Sensorial & & 2 & 1 & & 2 & & & Intuitivo \\
\hline Visual & 1 & 3 & & & & 1 & & Verbal \\
\hline Sequencial & & & 1 & & 1 & 3 & & Global \\
\hline
\end{tabular}

Ao analisar os resultados é possível observar que a maioria dos estudantes prefere aprender por meio de dados concretos, bem como possuem estilo moderadamente e/ou fortemente Visual além de moderadamente Global.

$\mathrm{Na}$ segunda fase foi realizada uma avaliação utilizando um questionário baseado no modelo de treinamento de Kirkpatrick (1994) contendo as seguintes afirmativas: Atenção - (1) O design da interface dos jogos é atraente; Relevância - (2) Ficou claro para mim como o conteúdo dos jogos está relacionado com coisas que eu já sabia; (3) Eu poderia relacionar o conteúdo dos jogos com coisas que já vi, fiz ou pensei; Satisfação - (4) Completar os exercícios dos jogos me deu um sentimento de realização; Desafio - (5) Eu gostei do jogo e não me senti ansioso ou entediado; Divertimento - (6) Eu jogaria estes jogos novamente; Conhecimento - (7) Depois dos jogos consigo lembrar de mais informações relacionadas ao tema; (8) Depois dos jogos consigo compreender melhor os temas apresentados nos jogos.

Ao observar a Tabela 2 é possível analisar os resultados obtidos com a aplicação do modelo. A partir da análise nota-se que apenas $20 \%$ dos alunos concordaram 
V Congresso Brasileiro de Informática na Educação (CBIE 2016)

Anais do XXII Workshop de Informática na Escola (WIE 2016)

totalmente que os objetos de aprendizagem utilizados nas aulas de Português e Matemática (jogos) possuíam características que prenderam a atenção dos mesmos. Acredita-se que esse resultado tem ligação com estilo de aprendizagem da maioria dos estudantes (Visual), pois como mencionado na etapa (SOE) os OA selecionados para a disciplina de Português não possuíam características visuais.

Tabela 2. Resultado da avaliação de treinamento

\begin{tabular}{|c|c|c|c|c|c|}
\hline & $\begin{array}{c}\text { Concordo } \\
\text { totalmente }\end{array}$ & $\begin{array}{c}\text { Concordo } \\
\text { em parte }\end{array}$ & $\begin{array}{c}\text { Não tenho } \\
\text { opinião }\end{array}$ & $\begin{array}{c}\text { Discordo } \\
\text { em parte }\end{array}$ & $\begin{array}{c}\text { Discordo } \\
\text { totalmente }\end{array}$ \\
\hline Atenção & $20 \%$ & $40 \%$ & - & $40 \%$ & - \\
\hline Relevância & $80 \%$ & $20 \%$ & - & - & - \\
\hline Satisfação & $40 \%$ & $40 \%$ & - & - & $20 \%$ \\
\hline Desafio & - & $60 \%$ & - & $40 \%$ & - \\
\hline Divertimento & - & $60 \%$ & - & - & $40 \%$ \\
\hline Conhecimento & $40 \%$ & $40 \%$ & - & $20 \%$ & - \\
\hline
\end{tabular}

Apesar disso, $80 \%$ dos estudantes concordaram que os artefatos educativos tinham ligação com os assuntos curriculares em questão. Ainda na Tabela 2 é possível observar que $80 \%$ concordaram totalmente ou em parte que os OA proporcionaram a eles satisfação ao término das atividades. Apesar da impossibilidade de generalização dos resultados devido a pequena amostra acredita-se que, em futuras aplicações da ABILSEN, pode ser importante o mapeamento dos estilos de aprendizagem dos estudantes antes da seleção dos OA de modo a aumentar as chances de aceitação dos OA pelos estudantes.

\subsection{Refinamento das Práticas}

Esta fase buscou analisar possíveis falhas cometidas na execução da abordagem. A partir de uma análise conjunta com os professores das disciplinas, conclui-se que a abordagem foi realizada com êxito. Tal conclusão encontra-se abarcada em alguns pontos, a saber: o feedback positivo dos alunos com relação à experiência interdisciplinar proposta pela abordagem; e a boa assimilação dos conceitos básicos de Computação por parte dos alunos, tendo em vista os artefatos educativos desenvolvidos por eles próprios.

\section{Considerações Finais}

Levando em consideração a necessidade da escola, enquanto formadora de cidadãos, moldar sua estrutura curricular de modo a contemplar dentre os saberes básicos o pensamento computacional, o presente artigo corrobora com essa inclusão ao analisar, revisar e aplicar a Abordagem para Inclusão do Licenciado em Computação no Ensino Básico.

$\mathrm{Na}$ fase de Análise da ABILSEN foram identificadas lacunas com relação à execução de algumas de suas etapas. Como revisão da abordagem foi proposta a inserção de perguntas de cunho reflexivo. Espera-se que o Licenciado em Computação, ao responder os questionamentos, consiga delinear critérios de modo a facilitar a execução da abordagem. A fim de verificar os impactos que as perguntas podem ter na organização da abordagem, a mesma foi aplicada em uma turma do Ensino Fundamental II. 
A pequena quantidade de alunos participantes resulta numa ameaça a validade externa. Assim, os resultados obtidos não podem ser generalizados para outros ambientes. Por outro lado, os resultados satisfatórios demonstram que as melhorias propostas tiveram influência positiva e direta na escolha da escola e na seleção de Objetos de Aprendizagem, além da decisão de incluir estratégias de gamificação nas etapas (AM) e (DOE) da abordagem. Por fim, projetam-se como trabalhos futuros a reaplicação da abordagem com uma amostra maior de estudantes, onde a seleção de objetos de aprendizagem e formação dos grupos na fase (DOE) levará em consideração inventários de estilos de aprendizagem.

\section{Referência}

Araújo, D. C.; Rodrigues, A. N.; Silva, C. V. A.; Soares, L. S. (2015) O Ensino de Computação na Educação Básica apoiado por Problemas: práticas de licenciandos em Computação. In: Anais do XXIII Workshop sobre Educação em Informática.

Castro, F. S. de, Fernandes, A. M. da R. (2013) Ambiente de Ensino de Quimíca Orgânica Baseado em Gamificação. In: Revista de Exatas e Tecnologia, v. 1, n. 4.

Felder, R. M. (2002) Author's Preface - June 2002. In: FELDER, R. M.; SILVERMAN, L. K. Learning and Learning and Teaching Styles in Engineering Education. Disponível em: <http://www.ncsu.edu/felder-public/Papers/LS1988.pdf>. Acesso em: Maio de 2015.

Felder, R. M.; Silverman, L. K. (1988) Learning and Teaching Styles in Engineering Education. Journal of Engineering Education, v. 78, n. 7, p. 674-68 Disponível em: $<$ http://www.ncsu.edu/felder-public/Learning_Styles.html $>$. Acesso em: Maio de 2015.

Fioro, R. et al. (2014). Uma experiência prática da inserção da robótica e seus benefícios como ferramenta educativa em escolas públicas. In: Anais do Simpósio Brasileiro de Informática na Educação.

Freire P. (1997) Pedagogia da Autonomia: saberes necessários a prática educativa. 9 ed. São Paulo: Paz e Terra.

Kirkpatrick, D. L. (1994) Evaluating training programs: the four levels. San Francisco: Berrett-Koehler.

Lopes, R. A.; Toda, A. M.; Brancher, J. d. (2015) Um estudo preliminar sobre elementos extrínsecos e instrínsecos do processo de Gamification. In: Revista Brasileira de Informática na Educação RBIE, v. 23, n. 3, pág. 165-173.

Neto, S. R. S.; Santos, H. R. M.; Santos, W. O. S. (2015) ABILSEN: Uma Abordagem para Inclusão do Licenciado em Computação no Ensino Básico. In: Anais do XXIII Workshop sobre Educação em Informática.

Sá, N. M. C. (2004) O lúdico na ciranda da vida adulta. Dissertação (mestrado) Universidade do Vale do Rio dos Sinos.

Scaico, P. D. et al. (2012) Um Relato de Experiências de Estagiários da Licenciatura em Computação com o Ensino de Computação para Crianças. In: Revista Novas Tecnologias - RENOTE, v.10, n.3. 